\title{
$\langle$ 无损检测〉
}

\section{基于红外无损检测的非金属材料粘贴缺陷识别}

\author{
牛 奕, 甘玲童, 马 云 \\ (武汉理工大学 安全与应急管理学院, 湖北 武汉 430070)
}

\begin{abstract}
摘要: 非金属材料粘贴结构的脱粘现象可影响其性能，使用红外无损检测技术可对粘贴缺陷进行有 效的识别。首先研究了基于红外无损检测技术的粘贴缺陷边界特征, 确定了使用温度梯度极值判断 粘贴缺陷边界位置的定量分析方法; 结合该特征, 采用了 Canny 边缘检测算法对数值模拟的粘贴缺 陷模型进行缺陷边界识别。同时使用该算法对实验数据进行识别, 针对识别结果出现的边界模糊、 噪点多等问题, 提出了篎选出所有 “疑似边界” 以保留 “弱边界” 的改进算法。结果表明, 改进后 的 Canny 算法能够提高红外无损检测粘贴缺陷的完整性和准确性。
\end{abstract}

关键词: 红外无损检测; 粘贴缺陷; 识别算法; 缺陷定量

中图分类号：TN215 文献标识码：A＼cjkstart文章编号：1001-8891(2020)04-0393-06

\section{Identification of Adhesive Defects of Non-metallic Materials Based on Infrared Non-destructive Testing}

\author{
NIU Yi, GAN Lingtong, MA Yun \\ (School of Safety Science and Emergency Management, Wuhan University of Technology, Wuhan 430070, China)
}

\begin{abstract}
The debonding of non-metallic materials can affect their performance, and infrared nondestructive testing can identify adhesive defects effectively. The boundary feature of adhesive defects is first investigated in this study based on infrared non-destructive testing; subsequently, a quantitative analysis method for identifying the boundary position of adhesive defects using the extreme value of a temperature gradient is obtained. Next, the Canny edge detection algorithm is used to identify the defect boundary of an adhesive defect model by numerical simulations and to identify experimental data simultaneously. For problems such as blurred boundaries and noise for recognition results, an improved algorithm for filtering out all "suspected boundaries" to preserve the "weak boundary" is proposed. The results show that the improved Canny algorithm can improve the integrity and accuracy of identifying adhesive defects from infrared non-destructive testing.
\end{abstract}

Key words: infrared nondestructive testing, adhesive defects, recognition algorithm, quantitative analysis of defects

\section{0 引言}

非金属材料的粘贴结构在工业生产、航空航天、 军事、建筑等领域具有广泛的应用性和诸多的优越 性 ${ }^{[1]}$, 但是粘贴界面容易出现脱粘、分层、夹杂等缺 陷, 进而降低系统的保温隔热性、耐久性和防火安全 性, 最终引发安全问题。因此, 对非金属材料的粘贴 缺陷进行无损检测具有重要意义。

近些年来应用广泛的红外无损技术与其他常规
无损检测方法相比, 具有适用范围广、非接触性、测 温范围广、结果直观可靠等 ${ }^{[2]}$ 优势。目前许多学者基 于红外无损检测技术, 对材料内部的缺陷进行探测, 对其进行定性定量分析, 同时提出各种算法以实现缺 陷的可视化。在缺陷的定性定量方面, M. Barus ${ }^{[3]}$ 等 通过对复合材料内部粘贴缺陷试样表面温度场的处 理, 验证了红外无损检测技术对检测复合材料脱粘缺 陷的可行性; 刘俊岩 ${ }^{[4]}$ 等建立热波在试件中传导的热电等效模型, 得到了缺陷深度和反射热波与入射热波 
相位差之间的关系，获得缺陷大小及位置信息; 王黎 明 ${ }^{[5]}$ 等运用红外热图和温差-时间对数曲线两种方式 识别缺陷, 缺陷识别精度达到 $0.4 \mathrm{~mm}$; Balageas ${ }^{[6]}$ 和 Holtmnn ${ }^{[7]}$ 等分别总结了脉冲法和锁相法在缺陷厚度、 类型、导热率等定性定量分析的应用。在缺陷识别及 提取方面, 汪子君 ${ }^{[8]}$ 等通过改进 OSTU 算法搜索最佳 阈值实现红外图像的缺陷分割; 梅林 ${ }^{[9]}$ 等提出基于遗 传算法优化求解的红外无损检测缺陷信息新方法; 王 涛等 ${ }^{[10]}$ 人将粒子群优化算法和二维最小误差分割算 法相结合, 能够在实现更低对比度的红外图像分割; 范春利 ${ }^{[11-12]}$ 等使用共轭梯度法都缺陷边界进行定量 识别, 数值算例证明了该算法的有效性; 此外, 还有 学者基于 $\mathrm{BP}$ 神经网络 ${ }^{[13]}$ 、小波变换 ${ }^{[14]}$ 等识别并提取 缺陷。缺陷的识别技术虽然已经取得了宝贵成果, 但 由于实验环境、技术制约等问题, 在实际工程应用中 仍然无法进行精确的缺陷定量识别。

本文研究了持续激励条件下非金属材料粘贴缺 陷的边界特征, 确定了对粘贴缺陷边界进行定量分析 的方法。根据边界特征结合了 Canny 边缘检测算法, 改进了算法的识别结果, 最终对粘贴缺陷边界几何特 征进行识别, 并标识出缺陷区域。实现对非金属材料 粘贴缺陷的定量检测并将缺陷可视化, 可为红外无损 检测的工程应用提供理论基础和技术指导。

\section{1 粘贴缺陷边界特征研究}

目前红外无损检测技术根据激励方式不同, 主要 分为脉冲激励红外无损技术、锁相激励红外无损技术 和持续激励红外无损技术。其中, 持续热激励方式相 较于其他热激励方式, 更适合热导系数较低且厚度较 大的非金属材料 ${ }^{[15]}$ 。本文采用持续热激励法研究粘贴 缺陷, 使用 CFD 流体分析软件研究在二维条件下的 缺陷边界特征, 并提出缺陷边界识别方法, 最后在三 维条件下验证方法的可靠性。

\section{1 二维数值模拟}

假设防腐材料片状的粘贴缺陷是由粘贴时胶水 中含有气泡导致, 则防腐材料表面在激励辐射热流作 用下温度上升, 而缺陷部位 (空气) 的热扩散系数要 远小于胶水, 导致该部分热量扩散较无缺陷部位缓 慢, 因此该部位对应的表面温度比周围温度较高。本 文为研究非金属材料粘贴缺陷边界的温度分布特征, 使用 CFD 流体分析软件, 假设基体材料为铁, 非金 属材料为 $\mathrm{PE}$, 两者之间使用胶水粘贴, 胶水的厚度 处处相等, 气泡假设由空气填满且位于试样的中心, 气泡的大小和高度即为缺陷的大小和程度。试件二维 模型及边界传热条件如图 1 所示。 4 种材料的密度、
导热系数、热容 ${ }^{[16]}$ 如表 1 所示。通过数值模拟计算, 图 2 给出稳定状态时不同缺陷半径 $R$ 下, 表面温度及 温度梯度的分布曲线，其中 $r$ 表示试件上表面各点与 对称轴的距离, $T$ 表示对应点的温度, $\mathrm{d} T / \mathrm{d} r$ 表示对应 点的温度梯度。

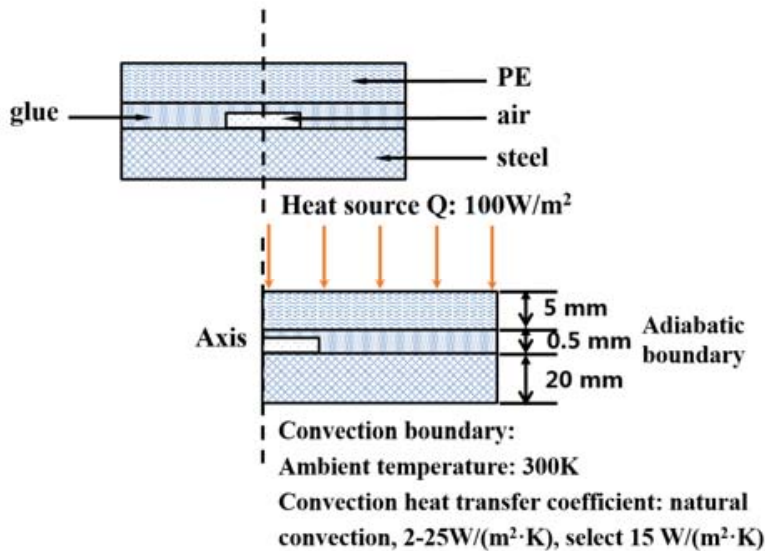

图 1 粘贴缺陷二维轴对称模型示意图

Fig.1 The diagram of two-dimensional axisymmetric model of paste defect

表 1 材料热物性参数

Table 1 Material thermal property parameters

\begin{tabular}{lccc}
\hline Material & $\begin{array}{c}\text { Density/ } \\
\left(\mathrm{kg} / \mathrm{m}^{3}\right)\end{array}$ & $\begin{array}{c}\text { Thermal } \\
\text { conductivity/ } \\
\left(\mathrm{W} /\left(\mathrm{m}^{2} \cdot \mathrm{K}\right)\right)\end{array}$ & $\begin{array}{c}\text { Heat } \\
\text { capacity/ } \\
(\mathrm{J} /(\mathrm{kg} \cdot \mathrm{K}))\end{array}$ \\
\hline Steel & 8030 & 16.27 & 502.48 \\
PE & 100 & 0.047 & 1380 \\
Glue & 1200 & 0.35 & 270 \\
Air & 1.225 & 0.0242 & 1006.43 \\
\hline
\end{tabular}

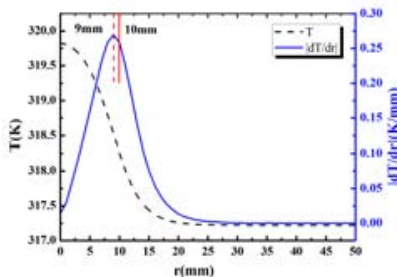

(a) $R=10 \mathrm{~mm}$

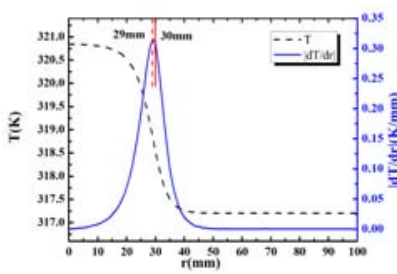

(c) $R=30 \mathrm{~mm}$

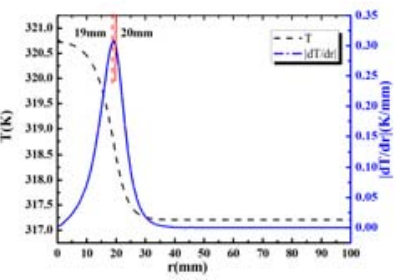

(b) $R=20 \mathrm{~mm}$
图 2 二维模型的表面温度及温度梯度分布曲线

Fig.2 Surface temperature and temperature gradient distribution curves of two-dimensional models 
从不同缺陷半径对应的温度分布曲线可以看出, 缺陷区域呈现高温状态, 缺陷边界处的温度快速下 降。根据表 2 缺陷边界的温度可得知, 随着缺陷半径 的增加, 缺陷边界对应的表面温度也随之升高, 这是 由于缺陷半径越大, 缺陷区域内积累热量越多, 试件 表面温度越高 ${ }^{[17]}$ 。此外, 粘贴材料的不同也会影响试 件表面温度 ${ }^{[18]}$ 。因此, 无法利用温度值作为阈值检测 出缺陷边界的准确位置, 需要找到更普遍的规律来识 别缺陷边界。

在粘贴缺陷的边界处, 由于空气的热扩散系数远 远小于胶水的热扩散系数, 因此热量由空气传向胶 水。根据导热的傅里叶定律, 在缺陷的边界热流密度 将达到峰值。因此, 可考虑通过计算温度梯度峰值位 置来确定缺陷边界。由温度梯度分布曲线可以看出, 温度梯度在缺陷边界位置附近达到极值。表 2 给出了 通过温度梯度极值确定的计算缺陷半径与实际缺陷 半径对比, 结果显示两者的相对误差较小, 因此验证 了使用温度梯度极值确定缺陷边界的可行性。

表 2 计算缺陷半径与实际缺陷半径的对比

Table 2 Comparison of simulated defect radius with actual defect radius

\begin{tabular}{cccc}
\hline $\begin{array}{c}\text { Actual } \\
\text { defect } \\
\text { radius } / \mathrm{mm}\end{array}$ & $\begin{array}{c}\text { Defect } \\
\text { boundary } \\
\text { temperature/K }\end{array}$ & $\begin{array}{c}\text { Simulated } \\
\text { defect } \\
\text { radius /mm }\end{array}$ & $\begin{array}{c}\text { Relative } \\
\text { error }\end{array}$ \\
\hline 10.0 & 318.26 & 9.0 & $-10.0 \%$ \\
20.0 & 318.47 & 19.0 & $-5.0 \%$ \\
30.0 & 318.53 & 29.0 & $-3.3 \%$ \\
40.0 & 318.58 & 39.0 & $-2.5 \%$ \\
\hline
\end{tabular}

\section{2 缺陷边界识别算法}

根据温度梯度在缺陷边界处达到极值这一特性, 准确地笁选出温度梯度极值部位即可识别边界位置。 由于 Canny边缘检测算法的原理是计算像素的梯度大 小及方向后采用非极大值抑制和双阈值来去除误检 点及保留漏检点 ${ }^{[19]}$, 这与篮选出温度梯度极值来确定 缺陷边界的过程一致, 因此考虑采用 Canny 边缘检测 算法对试件表面温度分布进行缺陷识别。

为验证使用 Canny 边缘检测算法可笁选出温度梯 度极值的可靠性, 本文首先利用 CFD 流体分析软件, 使用三维模型进行验证。建立含有底面为圆形及正方 形形状缺陷的三维模型, 如图 3(a)所示试件三维模型。 两种类型缺陷的底面尺寸如图 3(b)所示, 圆形直径 $\Phi$ 为 $40 \mathrm{~mm}$, 正方形边长 $L$ 为 $30 \mathrm{~mm}$ 。使用 CFD 流体 分析软件, 其边界条件及试件材料与二维模型一致, 得到如图 4 所示的试件表面温度分布云图, 结果显示 在缺陷对应的上表面存在局部的高温区域, 但无法准
确定位缺陷边界的位置。

对其进行一阶微分处理得到如图 5 所示的表面温 度梯度分布等高线图。由温度梯度等高线图可知, 缺 陷区域形成 “火山”状地形，在缺陷边界处形成 “山 脊”, 即温度梯度在缺陷边界处达到极值。对两种缺 陷的温度分布数据使用 Canny 算法进行检测, 得到如 图 6 所示的缺陷边界点集合。使用 Canny 算法得到的 缺陷边界所围成的区域面积与实际缺陷面积进行对 比，如表 3 所示。结果显示 Canny 边缘检测法检测出 的缺陷边界所围成的区域面积与实际缺陷区域面积 基本吻合。

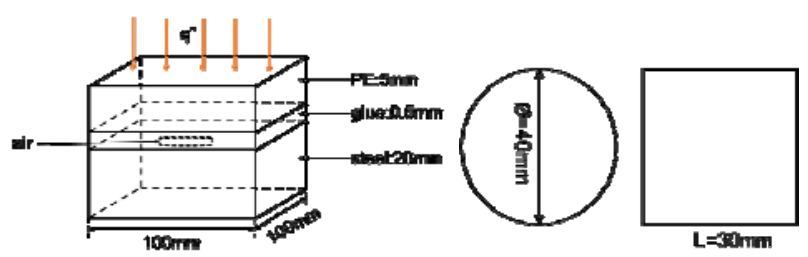

(a) 粘贴缺陷的三维模型

(a) 3D model of a test piece containing a paste defect

图 3 三维模型试件及其缺陷尺寸

Fig.3 3D model test piece and its defect size

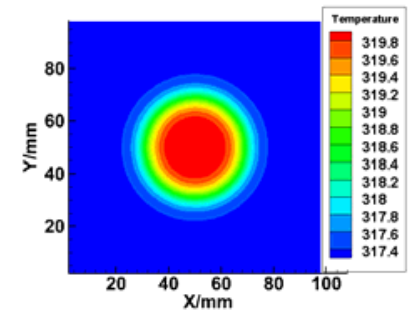

(a) 圆形缺陷

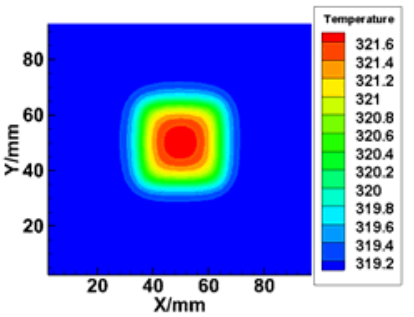

(b) 方形缺陷 (b) 缺陷尺寸

(b) Defect size
图 4 三维模型的表面温度分布云图 (b) Square defect

Fig.4 Surface temperature distribution of a three-dimensional model

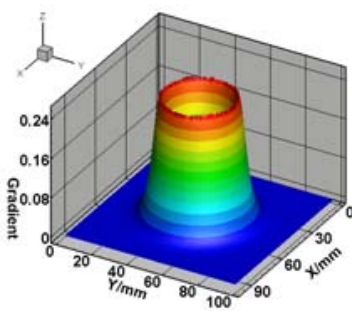

(a) 圆形缺陷

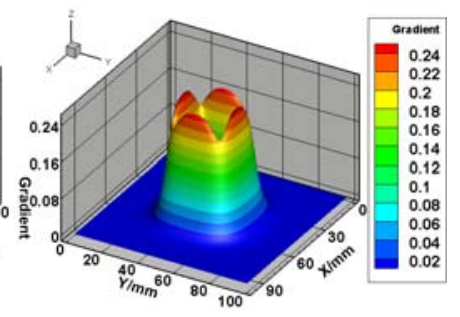

(b) 方形缺陷 (a) Circular defect

(b) Square defect

图 5 三维模型的温度梯度分布等高线图

Fig.5 Contour map of temperature gradient distribution of 3D model 


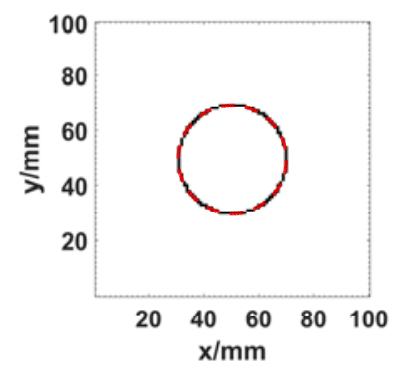

(a) 圆形缺陷

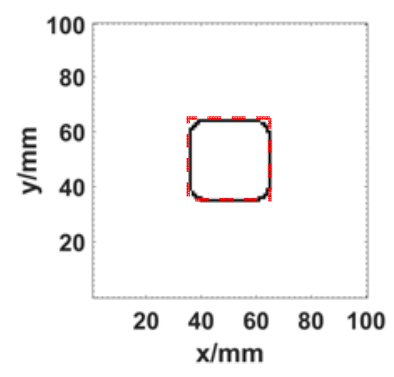

(b) 方形缺陷

(b) Square defect (a) Circular defect
外热图, 结果显示试件表面红外图具有 4 个明显的高 温区域, 但因受热不均、周围环境噪声等影响, 所采 集到的数据存在噪声较大、边缘模糊等问题。

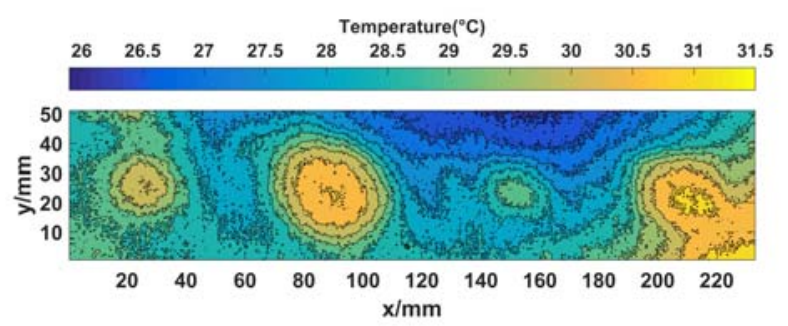

图 9 实验试件表面温度分布云图

Fig.9 Contour map of surface temperature distribution of experimental specimens

对试件表面温度分布数据使用高斯滤波进行降 噪处理后得到如图 10 所示的表面温度分布图。根据 前文得到使用温度梯度极值来确定缺陷边界的定量 分析方法, 对试件表面红外测温数据进行一阶微分处 理得到相应的温度梯度分布, 如图 11 所示, 采用 Canny 边缘检测算法对试样 4 块缺陷区域进行定量识 别, 其结果如图 12 所示。

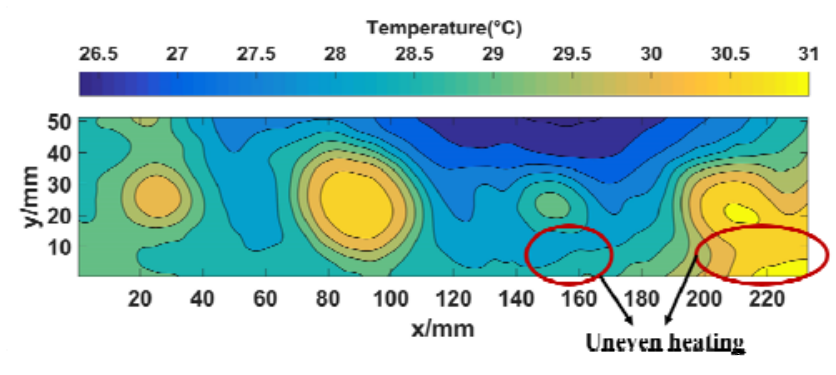

图 10 降噪处理后的试样表面温度云图

Fig.10 Contour map of the surface temperature of the sample after noise reduction treatment

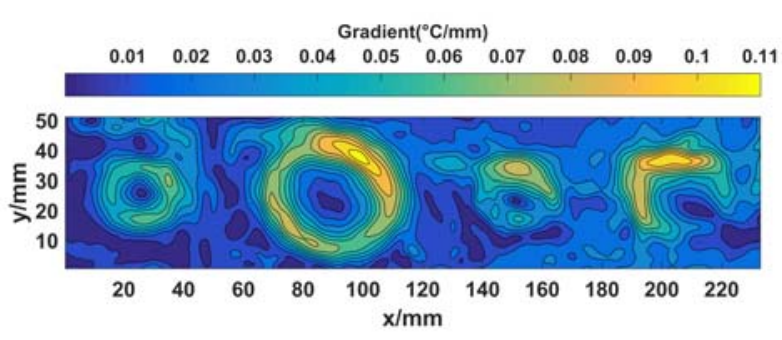

图 11 试样的表面温度梯度分布等高线图

Fig.11 Contour map of surface temperature gradient of sample

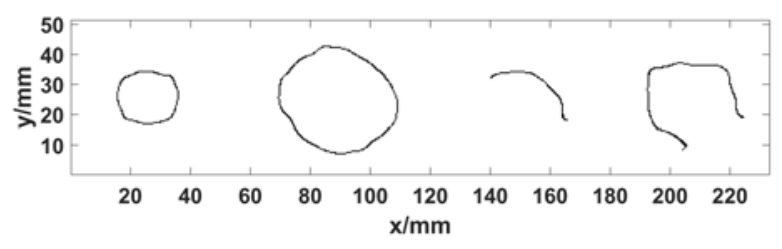

图 12 基于 Canny 算法的试样缺陷识别结果

Fig.12 Identification result of sample defects based on Canny algorithm
Fig.8 The diagram of a sample containing debonding defects

\section{2 实验结果分析}

试样在持续热激励 $150 \mathrm{~s}$ 后得到如图 9 所示的红 
结果显示使用 Canny 边缘检测算法识别的缺陷边 界与实际缺陷边界相比存在明显的误差, 第一个和第 二个缺陷的检测结果为完整、闭合的边界, 但第三个 和第四个缺陷边界只能检测到部分边界。这是由于缺 陷 3 和缺陷 4 受热不均匀, 造成缺陷边界处有局部高 温, 使检测到缺陷部分边界模糊, 如图 11 中标识出 的部分。而 Canny 边缘检测算法采用双阈值法, 从而 漏检了缺陷 3 和缺陷 4 部分边界模糊的 “弱边界”, 即温度梯度较小的部分, 导致检测到的缺陷 3、4 不 完整。

由以上分析可知, Canny 边缘检测法在数值模拟 理想条件下可识别出准确的缺陷边界, 但在实验条件 不理想时存在误差, 需要进一步改进算法得到较为理 想的识别效果。

\section{3 改进的缺陷边界识别及标识算法}

根据前文的数值模拟结果可知温度梯度在缺陷 边界达到极值, 因此缺陷边界处的像素点的温度梯度 值总会比领域至少一个方向的像素值大。利用这一结 论, 可设计算法篮选出所有 “疑似边界点” 集合, 具 体思路为: 将目标像素点 $P_{i, j}$ 与其领域水平、垂直、 斜 $45^{\circ}$ 四个方向上的像素点相比较, 若目标像素点的 温度梯度值大于任一方向上的两个像素点的温度梯 度值, 则认为该点为 “疑似边界点”。

对红外无损实验得到的试件表面温度梯度分布 数据进行该方法的篮选后得到如图 13 所示的 “疑似 边界点” 集合。从结果可知, 疑似边界点集合内包括 了 4 个完整的闭合缺陷, 且该形状与大小与实际缺陷 基本吻合。因此, 利用疑似边界点集合可对 Canny 算 法识别的缺陷边界进行补充, 从而得到完整、闭合的 缺陷边界。利用 “质心填充” 法, 从每个缺陷的质心 位置开始填充, 当遇到边界时停止填充, 得到最终识 别到的 4 个缺陷区域, 如图 14 所示。

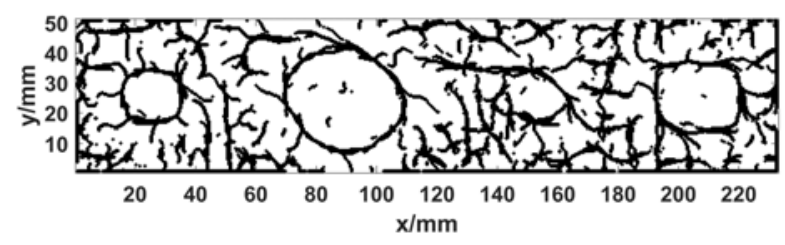

图 13 疑似边界点集合

Fig.13 Collection of points suspected of being borders

在计算缺陷面积时考虑了试样与入射光源存在 一定的夹角, 计算得到算法检测的缺陷面积与实际缺 陷面积，如表 4 所示。

根据计算结果可以看出, 检测缺陷面积与实际面 积相比存在一定的误差, 缺陷 1、2 存在较小误差,
但缺陷 3、4 误差明显偏大，这可能是由于缺陷 3、4 表面受热不均匀, 局部温度偏高从而产生的误差; 另 外一方面，由于缺陷 3、4 为正方形，其 4 个尖角部 位因热扩散导致其对应的温度分布呈现圆滑的形状， 导致检测到缺陷 3、4 的面积有部分损失。

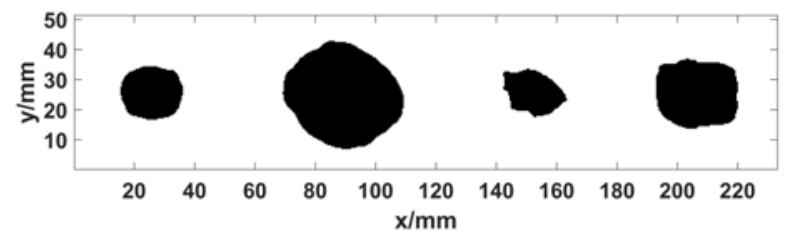

图 14 改进 Canny 算法得到的缺陷识别结果

Fig.14 Identification result of defects obtained by improved Canny algorithm

表 4 算法检测的缺陷面积与实际缺陷面积对比

Table 4 Comparison of defect area detected by algorithm and actual defect area

\begin{tabular}{cccc}
\hline $\begin{array}{c}\text { Defect } \\
\text { type }\end{array}$ & $\begin{array}{c}\text { Actual defect } \\
\text { area/cm }\end{array}$ & $\begin{array}{c}\text { Simulated defect } \\
\text { area } / \mathrm{cm}^{2}\end{array}$ & $\begin{array}{c}\text { Relative } \\
\text { error }\end{array}$ \\
\hline Defect 1 & 3.14 & 3.4 & $8.3 \%$ \\
Defect 2 & 12.56 & 11.81 & $-6.0 \%$ \\
Defect 3 & 4.0 & 2.63 & $-34.3 \%$ \\
Defect 4 & 9.0 & 6.07 & $-32.6 \%$ \\
\hline
\end{tabular}

\section{3 结论}

本文基于红外无损检测技术，在持续激励条件下 对粘贴缺陷试件进行数值模拟和实验验证, 研究了温 度梯度分布与缺陷边界的关系, 并设计了粘贴缺陷的 定量识别算法。主要结论如下:

1）通过建立二维及三维粘贴缺陷模型，在持续 激励条件下得到模型对应的上表面温度分布及温度 梯度分布，温度梯度在缺陷边界处达到极值;

2) 由于实验得到红外热图存在部分边界模糊、 噪声较大等问题, 对 Canny 算法进行改进后, 可补充 其缺损的缺陷边界，得到完整、闭合的缺陷边界。

\section{参考文献:}

[1] 刘颖蹈, 郭广平, 曾智, 等. 红外热像无损检测技术的发展历程、现状 和趋势 [J]. 无损检测, 2017, 39(8): 63-70.

LIU Yingwei, GUO Guangping, ZENG Zhi, et al. Development history, current situation and trend of infrared thermal image nondestructive testing technology[J]. Nondestructive Testing, 2017, 39(8): 63-70.

[2] 郑凯, 江海军, 陈力. 红外热波无损检测技术的研究现状与进展 [J]. 红外技术, 2018, 40(5): 401-411.

ZHENG Kai, JIANG Haijun, CHEN Li. Research status and development of infrared thermal wave nondestructive testing technology[J]. Infrared 
Technology, 2018, 40(5): 401-411.

[3] Barus M, Welemane $H$, Collombet $F$, et al. Bonded repair issues for composites: an investigation approach based on infrared thermography[J]. NDT \& E International, 2017, 85: 27-33.

[4] 刘俊岩, 戴景民, 王扬. 红外锁相法热波检测技术及缺陷深度测量 [J]. 光学精密工程, 2010, 18(1): 37-44.

LIU Junyan, DAI Jingmin, WANG Yang. Infrared phase-locking method for thermal wave detection and defect depth measurement[J]. Optics and Precision Engineering, 2010, 18(1): 37-44.

[5] 王黎明, 刘立帅, 梅红伟，等. 基于脉冲红外热波技术的支柱瓷绝缘 子无损检测方法[J]. 中国电机工程学报, 2017，37(24): 7359-7366, 7450 .

WANG Liming, LIU Lishuai, MEI Hongwei, et al. Non-destructive testing method for pillar porcelain insulator based on pulsed infrared thermal wave technology[J]. Proceedings of the CSEE, 2017, 37(24): 7359-7366, 7450 .

[6] Balageas D L. Thickness or diffusivity measurements from front face flash experiments using the TSR(thermoghic signal reconstruction) approach[C]//10th International Conference on Quantitative InfraRed Thermography, 2010: 873-880.

[7] Holtmnn N, Spiessberger C, Tleiter A, et al. Feature extraction from lock-in thermoghy phase images[C]//10th International Conference on Quantitative InfraRed Thermograph, 2010: 107-113.

[8] 汪子君, 邱俨睿, 杨宏霄, 等. 基于鲁棒 Otsu 的红外无损检测缺陷分 割算法[J]. 红外与激光工程, 2019, 48(2): 87-95.

WANG Zijun, QIU Yirui, YANG Hongwei, et al. Defect segmentation algorithm based on robust Otsu for infrared nondestructive testing[J]. Infrared and Laser Engineering, 2019, 48(2): 87-95.

[9] 梅林, 吴立德, 王裕文. 一种基于遗传算法的红外无损检测缺陷的新 方法[J]. 光学学报, 2002, 22(12): 1452-1456.

MEI Lin, WU Lide, WANG Yuwen. A new method for defects of infrared nondestructive testing based on genetic algorithm[J]. Acta Optica Sinica, 2002, 22(12): 1452-1456.

[10] 王涛, 陈凡胜, 苏晓锋. 二维最小误差分割在红外图像中的快速实现 [J]. 红外技术, 2016, 38(12): 1038-1041.

WANG Tao, CHEN Fansheng, SU Xiaofeng. Fast implementation of two-dimensional minimum error segmentation in infrared image[J]. Infrared Technology, 2016, 38(12): 1038-1041.

[11] 范春利, 孙丰瑞, 杨立. 边界形状的红外识别算法[J]. 化工学报, 2010, 61(11): 2783-2788.

FAN Chunli, SUN Fengrui, YANG Li. Infrared recognition algorithm for boundary shape[J]. Journal of Chemical Industry and Engineering, 2010,
61(11): 2783-2788.

[12] 范春利, 孙丰瑞, 杨立. 二维缺陷的定量识别技术研究 [J]. 热科学与 技术, 2008(3): 262-267.

FAN Chunli, SUN Fengrui, YANG Li. Study on quantitative recognition technology of two-dimensional defects[J]. Journal of Thermal Science and Technology, 2008(3): 262-267.

[13] 黄华. 基于 BP 神经网络的焊缝缺陷建模及其识别算法研究[D]. 西安: 西安石油大学, 2016.

HUANG Ye. Welding defects modeling and recognition algorithm researching based on Back-Propagation neural network[D]. Xi'an: Xi'an Shiyou University, 2016.

[14] 周鹏，徐科，刘顺华. 基于剪切波和小波特征融合的金属表面缺陷识 别方法[J]. 机械工程学报, 2015, 51(6): 98-103.

ZHOU Peng, XU Ke, LIU Shunhua. Metal surface defect recognition method based on shear wave and wavelet feature fusion[J]. Chinese Journal of Mechanical Engineering, 2015, 51(6): 98-103.

[15] 王冠. 持续激励红外热波无损检测系统数据处理软件实现与应用[D]. 北京: 首都师范大学, 2013 .

WANG Guan. Implementation and application of persistent excitation infrared thermal wave nondestructive testing system data-processing Software[D]. Beijing: Capital Normal University, 2013.

[16] 周辉, 钱美丽, 冯金秋, 等. 建筑材料热物理性能与数据手册 [M]. 北 京: 中国建筑工业出版社, 2010.

ZHOU Hui, QIAN Meili, FENG Jinqiu, et al. Handbook of Thermophysical Properties and Data for Building Materials[M]. Beijing: China Construction Industry Press, 2010.

[17] 牛奕, 马云, 李明明, 等. 非金属材料红外无损检测的建模和数值分 析[J]. 红外技术, 2019, 41(3): 214-219.

NIU Yi, MA Yun, LI Mingming, et al. Modeling and numerical analysis of infrared nondestructive testing of non-metallic materials[J]. Infrared Technology, 2019, 41(3): 214-219.

[18] 冯琪智. 基于红外热成像的复合材料无损检测研究 [D]. 成都：电子 科技大学, 2018 .

FENG Qizhi. Non-destructive testing of composite materials based on infrared thermography[D]. Chenngdu: University of Electronic Science and Technology of China, 2018.

[19] 许宏科, 秦严严, 陈会茹. 一种基于改进 Canny 的边缘检测算法 [J]. 红外技术, 2014, 36(3): 210-214.

XU Hongke, QIN Yanyan, CHEN Huiru. An edge detection algorithm based on improved canny[J]. Infrared Technology, 2014, 36(3): 210-214. 